\title{
Diffuse optical spectroscopic imaging for the investigation of human lactation physiology: a case study on mammary involution
}

Nienke Bosschaart
Anaïs Leproux
Ola Abdalsalam
Wen-Pin Chen
Christine E. McLaren
Bruce J. Tromberg
Thomas D. O'Sullivan 


\title{
Diffuse optical spectroscopic imaging for the investigation of human lactation physiology: a case study on mammary involution
}

\author{
Nienke Bosschaart, ${ }^{a, \star}$ Anaïs Leproux, ${ }^{b}$ Ola Abdalsalam, ${ }^{c}$ Wen-Pin Chen, ${ }^{d}$ Christine E. McLaren, ${ }^{e}$ \\ Bruce J. Tromberg, ${ }^{b}$ and Thomas D. O'Sullivan ${ }^{c}$ \\ aUniversity of Twente, Technical Medical Center, Biomedical Photonic Imaging Group, Enschede, The Netherlands \\ bUniversity of California Irvine, Beckman Laser Institute and Medical Clinic, Irvine, California, United States \\ 'University of Notre Dame, Department of Electrical Engineering, Notre Dame, Indiana, United States \\ dUniversity of California Irvine, Chao Family Comprehensive Cancer Center, Orange, California, United States \\ eUniversity of California Irvine, Department of Epidemiology, Irvine, California, United States
}

\begin{abstract}
Relatively few imaging and sensing technologies are employed to study human lactation physiology. In particular, human mammary development during pregnancy as well as mammary involution after lactation have been poorly described, despite their importance for breast cancer diagnosis and treatment during these phases. Our case study shows the potential of diffuse optical spectroscopic imaging (DOSI) to uniquely study the spatiotemporal changes in mammary tissue composition during the involution of the lactating breast toward its pre-pregnant state. At nine time intervals over a period of eight months after the cessation of breastfeeding, we reconstructed 2-D maps of mammary water content, lipid content, total hemoglobin (THb) concentration, oxygen saturation $\left(\mathrm{StO}_{2}\right)$, and tissue optical scattering. Mammary lipid content in the nonareolar region showed a significant relative increase of $59 \%$, whereas water content and $\mathrm{THb}$ concentration showed a significant relative decrease of $50 \%$ and $48 \%$, respectively. Significant changes were also found in $\mathrm{StO}_{2}$ and tissue optical scattering. Our findings are consistent with the gradual replacement of fibroglandular tissue by adipose tissue and vascular regression during mammary involution. Moreover, our data provide unique insight into the dynamics of breast tissue composition and demonstrate the effectiveness of DOSI as a technique to study human lactation physiology. () The Authors. Published by SPIE under a Creative Commons Attribution 4.0 Unported License. Distribution or reproduction of this work in whole or in part requires full attribution of the original publication, including its DOI. [DOI: 10.1117/1 .JBO.24.5.056006]
\end{abstract}

Keywords: lactation; involution; breast; diffuse optical spectroscopy; imaging.

Paper 190095R received Apr. 1, 2019; accepted for publication May 3, 2019; published online May 23, 2019.

\section{Introduction}

Due to the benefits of breastfeeding for infants, mothers, and societies, it is estimated that the deaths of 823,000 children and 20,000 mothers can be averted annually through universal breastfeeding. ${ }^{1}$ However, despite the advice of the World Health Organization, only $40 \%$ of infants are exclusively breastfed until the age of six months. ${ }^{2}$ The most important reasons for the early cessation of breastfeeding are the perception of insufficient milk supply and other breastfeeding problems, such as pain. ${ }^{3}$ More knowledge on the physiology of lactation can contribute to a better understanding of milk synthesis, milk transfer to the infant, as well as the development and management of breastfeeding problems. ${ }^{4}$ In turn, this will lead to improved guidelines and approaches for the support of mothers who experience breastfeeding problems.

Interestingly, few imaging and sensing modalities have been exploited to investigate lactation physiology in the human mammary gland in vivo. In the past 15 years, ultrasound imaging has played an important role in (re)defining the anatomy and physiology of the lactating breast. ${ }^{5,6}$ The other major noninvasive breast imaging modalities - magnetic resonance imaging (MRI) and mammography—have also been applied to study the human

*Address all correspondence to Nienke Bosschaart, E-mail: n.bosschaart@ utwente.nl lactating breast, but most often in the context of image interpretation in pregnancy-associated breast cancer ${ }^{7-9}$ and only rarely to study lactation physiology itself. ${ }^{10}$

In this study, we exploit a breast imaging modality that is established in the field of biomedical optics, but new in the field of lactation research: diffuse optical spectroscopic imaging (DOSI). Compared to other imaging modalities, DOSI provides unique insights into the spatial distribution of lipid, water, and hemoglobin concentrations in tissue without the injection of contrast agents. DOSI further differentiates between tissue types through the quantification of optical tissue scattering, which is related to the size and distribution of microscopic tissue structures such as collagen fibers. So far, DOSI has been applied extensively to study breast cancer. ${ }^{11-15}$ Here we illustrate the potential of DOSI for research on lactation physiology by presenting a case study on imaging the involution of the lactating breast after the cessation of breastfeeding. As currently no data are available on the time-resolved changes in tissue composition during mammary involution, this will provide a deeper understanding on the physiology behind mammary involution, as well as the total duration of the involution process. These new insights into the dynamics of mammary tissue composition may also be important for breast cancer diagnosis and treatment in the lactating breast, because mammary tissue composition affects the visualization of malignant lesions ${ }^{7-9}$ and may also affect the pharmacokinetics of chemotherapeutic agents. ${ }^{16}$ 


\subsection{Involution of the Lactating Mammary Gland}

Animal studies have demonstrated that the involution of the lactating mammary gland is a two-phase process. ${ }^{17}$ The first phase is reversible and involves extensive cell death of the milk-producing secretory epithelium in the alveoli. During this phase, the suckling of the mother's offspring can reinitiate lactation. In the second phase, the mammary tissue is remodeled, which involves the breakdown of extracellular matrix, remodeling of blood vessels, ${ }^{18}$ and the re-emergence of lipid-filled adipocytes. ${ }^{17}$ MRI data from human volunteers have confirmed that significant changes occur during mammary involution in fibroglandular tissue content, as well as milk duct diameter. ${ }^{10}$ This process is also accompanied with changes in human milk protein, lactose, chloride, and sodium concentrations. ${ }^{19}$ The second involution phase is irreversible and returns the breast to a pre-pregnant state, albeit that epigenetic changes in the mammary tissue can facilitate lactation in subsequent reproductive cycles. ${ }^{20}$ In mice, the duration of the first involution phase is $\sim 48 \mathrm{~h}$, whereas that of the second phase is $\sim 72$ to $144 \mathrm{~h} .{ }^{17}$ In larger mammals, the total duration of the involution process may vary from several weeks to several months. ${ }^{21,22}$ To our knowledge, the total duration of the involution of the human lactating breast has not yet been assessed objectively in the literature.

\subsection{Hypothesis and Objectives}

On the macroscopic scale that is evaluated by DOSI, we expect several changes to occur in human mammary tissue composition during the involution process. Due to the replacement of fibroglandular tissue by adipose tissue, we hypothesize that mammary water content will decrease, and lipid content will increase during the involution process. ${ }^{17}$ We further hypothesize that mammary blood content decreases, due to vascular regression. ${ }^{18}$ The objective of this study was, therefore, to investigate whether DOSI can be employed to evaluate these involution related changes in mammary tissue composition, as well as the changes in oxygen saturation $\left(\mathrm{StO}_{2}\right)$ and the light scattering properties of tissue. Furthermore, we investigated whether we can use these changes in tissue composition to estimate the currently unreported time-dependence and duration of the mammary involution process.

\section{Materials and Methods}

\subsection{Participant}

The participant in this case study was a 37-year-old woman, who was breastfeeding her first child unilaterally with her left breast. Four years prior to this study, the participant was treated for an early stage high-grade invasive ductal carcinoma of the right (contralateral) breast. Treatment consisted of unilateral mastectomy and adjuvant chemotherapy with docetaxel, carboplatin, and trastuzumab.

After a lactation period of 14 months, breastfeeding was ceased and the DOSI measurements were started. Data were acquired between June 2015 and February 2016, over a total time lapse of 237 days ( 8 months). During the first 104 days (3.5 months), the breast was scanned with DOSI every 1 to 3 weeks. One more DOSI scan was taken at 237 days, resulting in a total of 9 DOSI scans in time. Briefly after this last DOSI scan, the participant discovered that she was pregnant with her second child and the study was halted. The participant did not breastfeed or extract any milk during the study period. At the time of cessation, the participant had a large, palpable duct in her lactating breast, which did not have a suspicious appearance under ultrasonography and MRI. As involution continued, the palpable duct gradually became less noticeable.

Ethical approval for this study was obtained from the Institutional Review Board of the University of California, Irvine (\#1995-563), and the participant gave written informed consent prior to the study.

\subsection{Diffuse Optical Spectroscopic Imaging}

Instrumentation. DOSI measurements were performed with a system that combines frequency domain photon migration (FDPM) and broadband near infrared (NIR: 650 to $1000 \mathrm{~nm}$ ) spectroscopy for quantitative, model-based measurements of tissue absorption and scattering properties and recovery of tissue oxy-hemoglobin $\left(\mathrm{ctHbO}_{2}\right)$, deoxy-hemoglobin (ctHHb), water $\left(\mathrm{ctH}_{2} \mathrm{O}\right)$, and lipid. ${ }^{23}$ The custom-built DOSI instrument utilized an FDPM module with fiber-coupled, modulated (50 to $500 \mathrm{MHz})$ laser diode sources at four wavelengths $(660,690$, 780 , and $830 \mathrm{~nm}$ ) to illuminate the breast. FDPM light was collected with a temperature-controlled avalanche photodiode sensor (1-mm diameter active area), built in to the handheld probe that was placed in direct contact with the skin during data acquisition. The broadband NIR component of the system consisted of a fiber-coupled white light source and spectrometer to measure continuous-wave reflectance. The separation between the light sources and detectors was $28 \mathrm{~mm}$, which corresponds to $\sim 10$ to $15 \mathrm{~mm}$ of depth sensitivity. Standardized procedures were developed for data collection and to monitor instrument performance. Two sets of three calibration measurements on two custom-fabricated solid breast-tissue simulating phantoms (Institut National d'Optique, Quebec, Canada; and UC Irvine Beckman Laser Institute, California) and on one reflectance standard (SRS-99-020, Labsphere Inc.) were performed before and after each subject measurement. These measurements were used for determining the instrument response-function, and monitoring instrument performance over the duration of the study. ${ }^{24}$

Spectroscopy. The approach to DOSI data acquisition and analysis has been previously described ${ }^{23}$ and is briefly summarized here. Model-based fits of DOSI data were used to retrieve the wavelength resolved tissue absorption coefficient $\mu_{a}(\lambda)$ and reduced scattering coefficient $\mu_{s}^{\prime}(\lambda)$. Values for water, ctHHb, $\mathrm{ctH}_{2} \mathrm{O}$, and bulk lipid were calculated by fitting a linear combination of their known molar extinction coefficient spectra to the tissue absorption coefficient values. From these quantities, the total hemoglobin $(\mathrm{THb})$ concentration $(\mathrm{THb}=\mathrm{ctHHb}+$ ctHbO $\left.{ }_{2}\right)$ and percent $\mathrm{StO}_{2}\left(\mathrm{StO}_{2}=\mathrm{ctHbO} / \mathrm{THb}\right)$ were calculated. Optical scattering was characterized by parameterizing the reduced scattering spectrum as $\mu_{s}^{\prime}=a\left(\lambda / \lambda_{0}\right)^{-b}$, where $a$ is a scattering prefactor that indicates the reduced scattering coefficient at $\lambda_{0}=500 \mathrm{~nm}$ and $b$ is the scattering power, which describes the wavelength dependence of scattering.

Imaging procedure. DOSI measurements were performed using a standard protocol. The subject was measured in a supine or reclining position [Fig. 1(a)]. The handheld DOSI probe was placed against the breast tissue, and sequential measurements were recorded in a rectangular grid pattern using $10-\mathrm{mm}$ spacing. Each measurement took $\sim 3 \mathrm{~s}$. The dimension of the grid was $9 \times 11 \mathrm{~cm}^{2}$, resulting in 120 measurement locations [Fig. 1(b)]. The grid size was chosen to fully capture the breast. For consecutive DOSI visits, individual static landmarks such as 
(a)



(b)

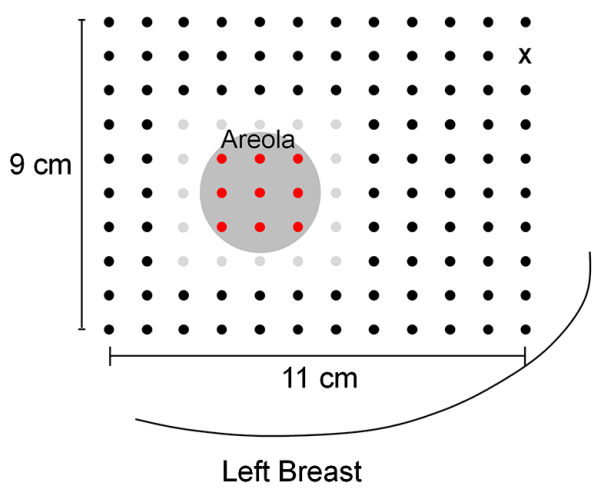

(c)

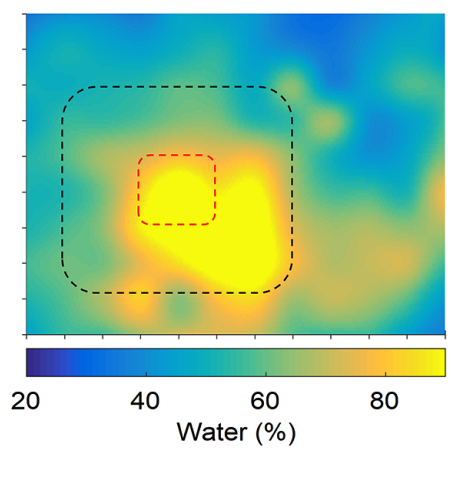

Fig. 1 Overview of DOSI. (a) Handheld DOSI probe and measurement setting for breast imaging demonstrated by researchers. (b) Measurement grid on the breast. Red and black dots denote the ROI for the areola, and nonareolar region, respectively. The gray dots account for the variability in areola size during the involution process and were not included in the analysis of the median DOSI parameters. The " $x$ " denotes the data point that was excluded from the analysis due to poor probe contact during one of the measurements. (c) Example of a resulting 2-D map for the water distribution in the breast, with the same spatial dimensions as figure (b). The dashed red and black lines represent the outer boundaries of the ROls for the areolar and nonareolar region, respectively. The areolar ROI is smaller than the actual size of the areola for this particular measurement (day 19).

moles or freckles were recorded on a transparency sheet and provided a fixed reference to coregister the imaging grid at follow-up DOSI sessions.

Image analysis. After initial processing, DOSI data (chromophore concentrations and scattering parameters) from each session were plotted using grid coordinates and heatmap functions in MATLAB. Cubic spline interpolation was used to account for 10-mm spacing of grid points, resulting in colored 2-D maps for water, lipid, $\mathrm{THb}, \mathrm{StO}_{2}$, scattering power, and scattering prefactor. An example for a 2-D water distribution map is given in Fig. 1(c).

After checking for normality, the median values of the optical property spectra and resulting DOSI parameters were calculated within both the areola (9 points) and nonareolar region (94 points), to evaluate their dynamics over the course of the involution process. Areola size was defined as the pigmented region that the DOSI operator recorded on the transparency sheet at day 0 . Although the DOSI operator did not record the time dependency in areola size, subsurface DOSI measurements suggested that areola size decreased during the involution process. Therefore, a consistent analysis was achieved by defining conservative constant region of interest (ROI) for the areola region and nonareolar region. To account for a potential reduction in areola size, the DOSI areola ROI was defined as $\sim 2-\mathrm{cm}$ diameter less than the visually recorded areola size at day 0 [red dots, Fig. 1(b)]. The nonareolar ROI was defined as the nonareola size at day 0 [black dots, Fig. 1(b)]. Grid points outside these ROIs [gray dots, Fig. 1(b)] were not included in the analysis of the median DOSI parameters to account for possible reduction in areola size over time. One point was excluded from the nonareolar region at all time points, due to poor data quality at a single-measurement session, which was caused by poor probe contact [indicated by a the " $x$ " in Fig. 1(b)].

\subsection{Statistical Analysis}

For each DOSI parameter, descriptive statistics of measurements such as median and percentiles were calculated by measurement day and used to construct Figs. 4(a)-4(f). To evaluate whether the DOSI parameters changed significantly in time, we analyzed the relative marginal effect for each DOSI parameter at each measurement day. ${ }^{25}$ This nonparametric analysis is more commonly used to determine whether a single group of homogeneous subjects experiences a change in some measurable parameter as a function of time. In this case, each "subject" is regarded as a single-spatial point on the breast. Due to the small sample size and non-normality of the DOSI parameters, this nonparametric analysis of longitudinal data in factorial experiments with repeated measures assumes that all spatial points were independent. Within each DOSI parameter, all measurements were ranked $\left(R_{i s}\right)$, where $i$ denotes the spatial point and $s$ denotes the day. The mean of ranks over spatial points was calculated by measurement day $\left(\bar{R}_{. s}\right)$. With $p_{s}$ representing the relative marginal effect at day $s$, the estimated relative marginal effect at day $s$ was calculated by $\hat{p}_{s}=\frac{1}{N} \times\left(\bar{R}_{. s}-\frac{1}{2}\right)$, where $N$ is the total number of measurements. A relative marginal effect $\hat{p}_{s}<\frac{1}{2}$ means that the experimental results at day $s$ tend to take on smaller values compared to the results at all days. The smaller the value of $p_{s}$, the stronger is the tendency to smaller values and vice versa. ${ }^{25}$ The estimated relative marginal effects and 95\% confidence intervals were used to construct Figs. 4(g)-4(1). We hypothesized that there was a measurement day effect (i.e., at least one difference in the estimated relative marginal effects across measurement days). All of the aforementioned procedures were performed in parallel and separately for both the areola and nonareolar region.

\section{Results}

Figure 2 illustrates the change in the DOSI-derived median absorption and reduced scattering spectra for both the areola and nonareolar region. For clarity, only four out of nine measurement days are shown. During the involution process, we can observe a decrease in the overall absorption spectra for both the ROIs. This can be ascribed to the variation in the individual contributions of the chromophores to these spectra over time. Note that the error bars (interquartile range of the data) are larger for the areola than for the nonareolar region, due to a smaller number of grid points that was used to calculate the median optical 


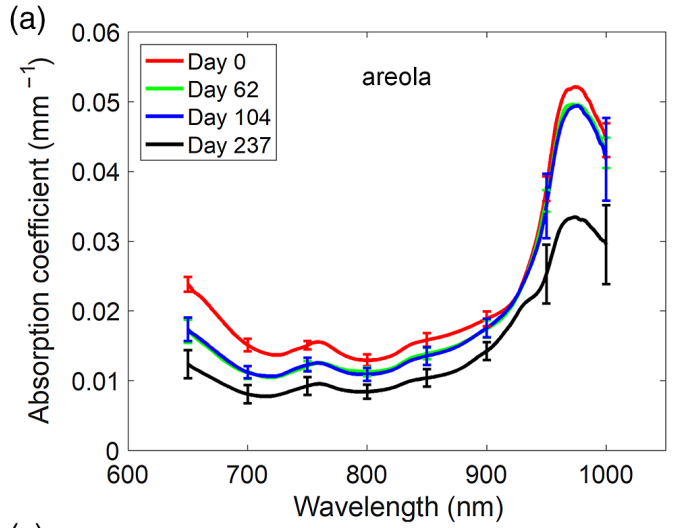

(c)

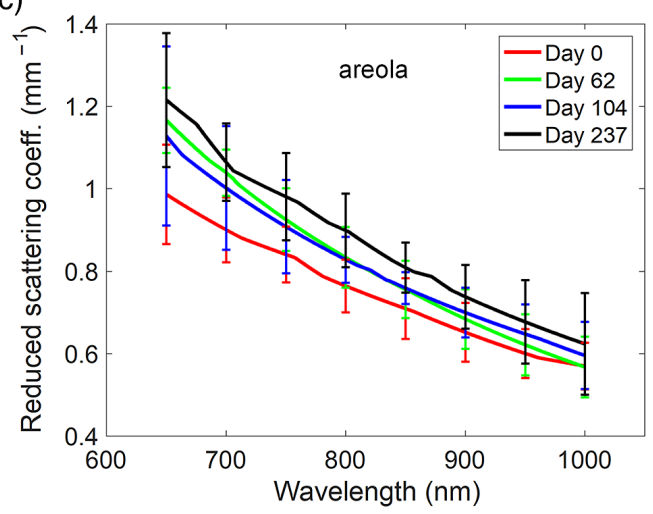

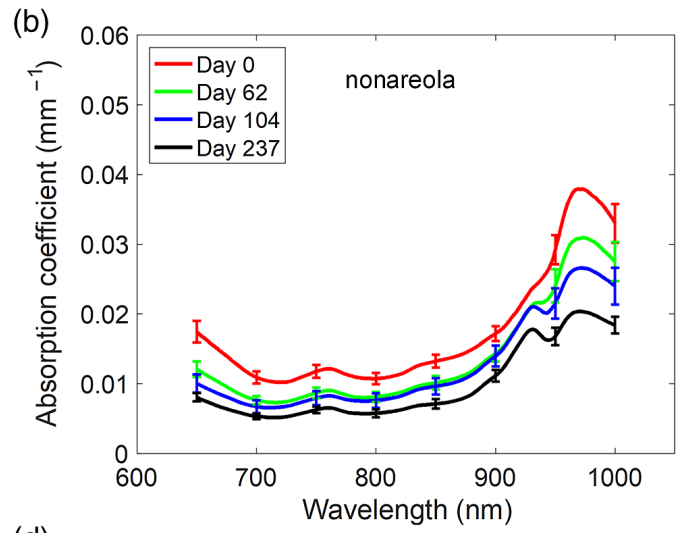

(d)

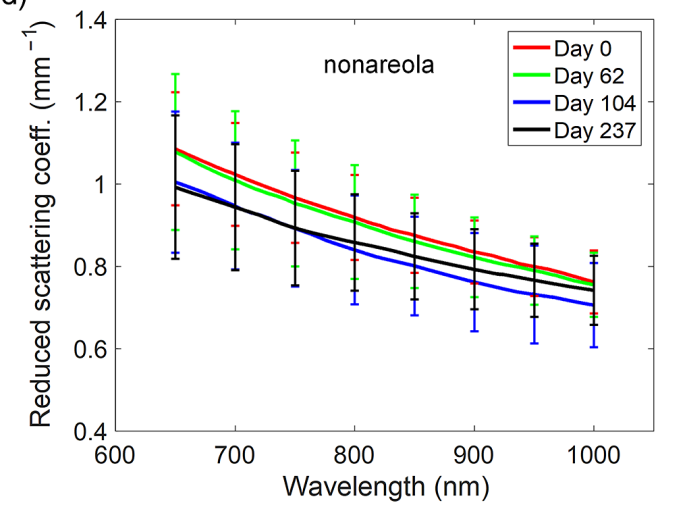

Fig. 2 Median optical property spectra. For clarity, only four out of nine measurement days are shown: (a) median absorption coefficient spectrum in the areola, (b) nonareolar region, (c) median reduced scattering coefficient spectrum in the areola, and (d) nonareolar region. Error bars represent the interquartile range of the data.

property spectra. Although the interquartile ranges of the reduced scattering coefficient spectra overlap, an increasing trend during the involution process can be observed for $\mu_{s}^{\prime}(\lambda)$ in the areola and a decreasing trend in the nonareolar region.

The spatial distribution maps for water, lipid, $\mathrm{THb}, \mathrm{StO}_{2}$, scattering power, and scattering prefactor are shown in Fig. 3. In addition to their time-dependency, most DOSI parameters differ between the areola and nonareolar region, which is in agreement with our previous findings. ${ }^{14}$ This spatial distribution of DOSI parameters suggests that the areolar diameter decreases in size during the involution process, which accounts for a factor 2.4 (ratio of the full-width half-maxima of the horizontal cross section through the water map at the position of the nipple between day 0 and day 237). Except for the final imaging session (day 237), the large palpable duct is characterized by an elevated water content and decreased lipid content at grid locations ( $x=+60$ to $70 \mathrm{~mm}, y=0$ to $+30 \mathrm{~mm}$ ).

To further investigate the changes of the DOSI parameters during the involution process, we calculated their median value and percentiles by measurement day and plotted them as a function of time for both the areola and nonareolar region [Figs. 4(a)-4(f)]. Based on the nonparametric statistical analyses, we concluded that there was a main measurement day effect with a $p$ value $<0.05$ for each DOSI parameter within each region. Hence, all DOSI parameters have time dependence after the cessation of breastfeeding [Figs. 4(g)-4(1)]. Although water content and $\mathrm{THb}$ concentration decrease as a function of time, lipid content and $\mathrm{StO}_{2}$ increase during the involution process. These relative changes are similar for both the areola and nonareolar region. The optical scattering changes are more complex. Although the nonareolar region shows a significant increase in scattering power and a decrease in scattering prefactor, the changes are less certain in the areola region due to the smaller number of grid points.

\section{Discussion}

In this study, we used DOSI to monitor the long-term changes in breast composition during mammary involution following breastfeeding. As hypothesized in the introduction, we measured a significant decrease in water content, as well as a significant increase in lipid content during the involution process. Both DOSI parameters changed at approximately the same rate, with relative changes in the nonareolar region of $50 \%$ in median water content and $59 \%$ in median lipid content between day 0 and day 237 (Fig. 4). These findings can be explained by the gradual replacement of fibroglandular tissue (high water, low lipid content) by adipose tissue (low water, high lipid content) during mammary tissue remodeling. ${ }^{26}$ The total change in water content between day 0 and day 237 is comparable to the change in fibroglandular tissue content between lactating and postweaning mammary tissue. ${ }^{10}$

Also the $\mathrm{THb}$ concentration decreased significantly with $48 \%$ in the nonareolar region between day 0 and day 237 , at a rate that is comparable to the changes in lipid and water content (Fig. 4). This decrease in THb concentration implies a decrease in mammary blood volume, which can be ascribed to vascular regression during mammary involution. ${ }^{18}$ Previously, animal studies have demonstrated that the hemoglobin 

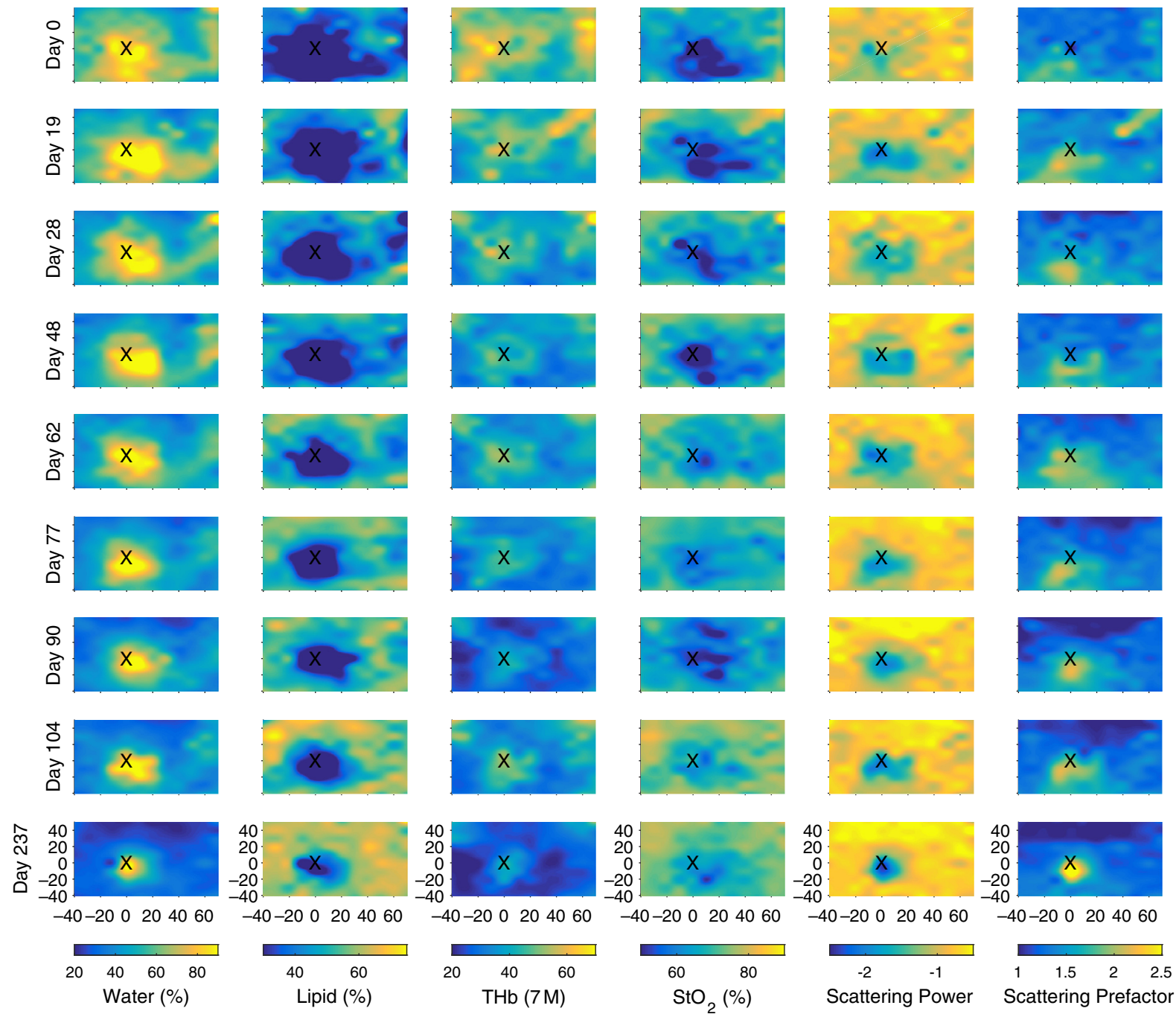

Fig. 3 Spatiotemporal changes in mammary tissue composition during involution. For the nine DOSI scans in time, the 2-D maps for the spatial distribution of water, lipid, $\mathrm{THb}, \mathrm{StO}_{2}$, scattering power, and scattering prefactor are shown. The axes of the 2-D maps denote the spatial position at the breast in $\mathrm{mm}$. The $\mathrm{X}$ marks the position of the nipple. Day 0 indicates the initiation of the cessation of breastfeeding.

concentration in blood increases during pregnancy, decreases during lactation, ${ }^{27}$ and increases again after lactation is ceased. ${ }^{28}$ As the measured tissue concentration of THb by DOSI depends both on vascular density and the hemoglobin concentration in whole blood, the observed decrease in $\mathrm{THb}$ may, therefore, underestimate mammary vascular density if the whole blood hemoglobin concentration increases during mammary involution. With only $11 \%$ difference in $\mathrm{StO}_{2}$, the nonareolar region between day 0 and day 237, these more modest changes in $\mathrm{StO}_{2}$ during the involution process are supported by similar $\mathrm{StO}_{2}$ levels between fibroglandular and adipose tissue in the breast. $^{26}$

The median scattering power and scattering prefactor in the nonareolar region changed with $20 \%$ and $11 \%$, respectively between day 0 and day 237. These modest, but significant changes can be explained by a difference in the microscopic structure (i.e., particle density and size) between fibroglandular and adipose tissue, which give rise to different scattering properties between both tissue types. ${ }^{26}$

The areolar region differed from the nonareolar region with an elevated water content, $\mathrm{THb}$ concentration, and scattering prefactor, as well as a decreased lipid content, $\mathrm{StO}_{2}$ level, and scattering power. These differences are consistent with the anatomy and physiology of the areola, which has an elevated concentration of lactiferous ducts that are supported by highly vascularized connective tissue. ${ }^{29}$ In contrast to the other DOSI parameters, the changes in scattering power and prefactor in the areolar region were not similar to those in the nonareolar region. An explanation for this observation can be that the high concentration of lactiferous ducts in the areola may remodel in a different manner during involution than the more lobule rich tissue of the nonareolar region. The areolar diameter at day 237 was approximately a factor 2.4 smaller compared to day 0 . Since areola size increases in a similar manner during pregnancy, ${ }^{30}$ it is, therefore, likely that the areola has returned to its (or close to) pre-pregnant state during the involution process.

\subsection{Study Limitations}

Since some of the DOSI parameters are still significantly different between day 237 and the preceding measurement at day 104 (nonareolar region), we cannot draw any definite conclusions 

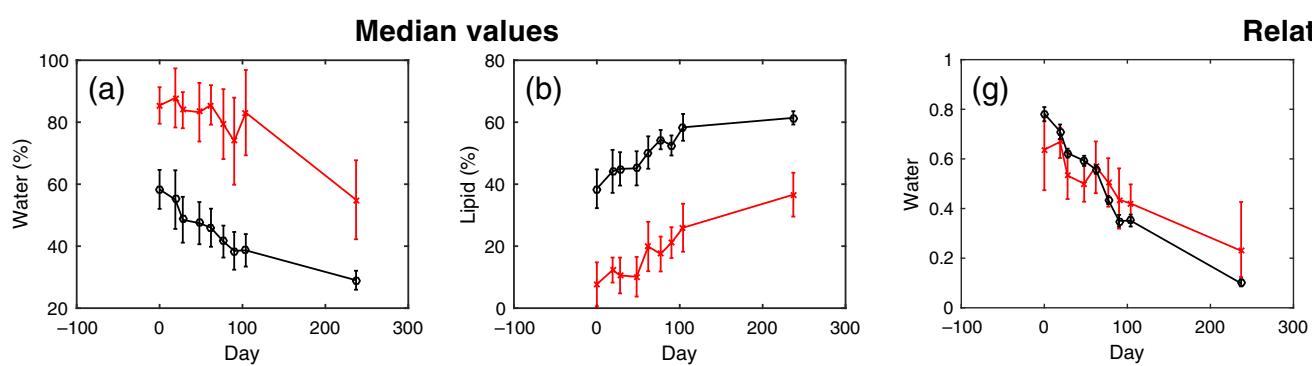

Relative effect
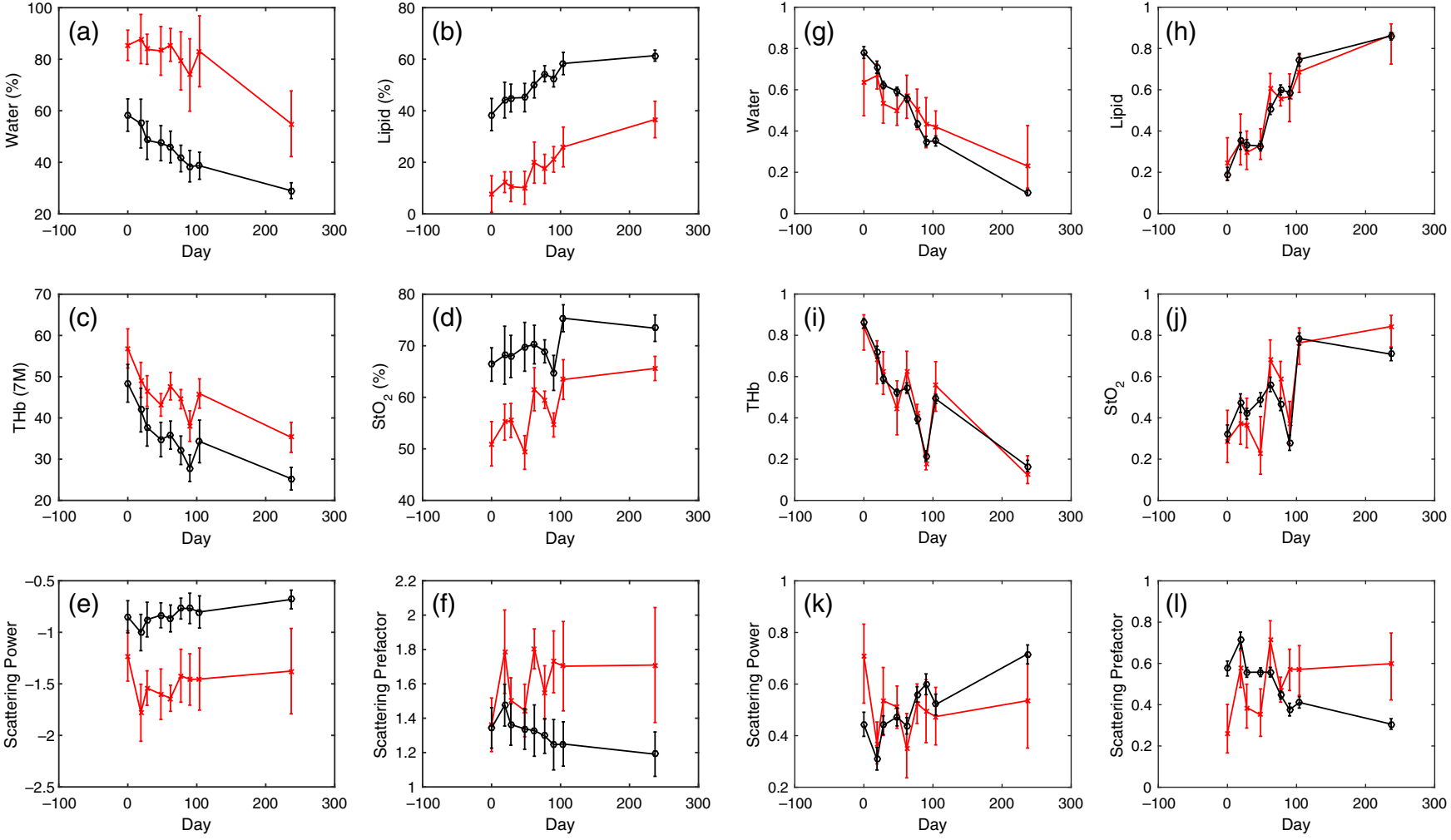

$\longrightarrow$ A- Areolar region
$\ldots$ Nonareolar region

Fig. 4 Median DOSI parameters and relative effect versus time during involution. Median values across the areolar and nonareolar are presented for (a) water content, (b) lipid content, (c) THb concentration, (d) $\mathrm{StO}_{2}$, (e) scattering power, and (f) scattering prefactor. Error bars in graphs (a)-(f) represent the interquartile range of the data. From our statistical analysis, the relative effect of the change in all DOSI parameters during involution is presented in graphs (g)-(l), where the error bars represent the $95 \%$ confidence intervals of the analysis.

about whether the complete involution process has been captured with this study. Nevertheless, the median water, lipid, $\mathrm{THb}$, and $\mathrm{StO}_{2}$ values in the nonareolar region at day 237 are comparable to those found in healthy, premenopausal breasts. ${ }^{14}$

Hormonal fluctuations during the menstrual cycle can cause significant fluctuations in the DOSI parameters, which may explain some of the variability in the DOSI parameters versus time in Fig. 4. ${ }^{31,32}$ The probing depth by our DOSI method inside breast tissue is $\sim 10$ to $15 \mathrm{~mm}$, so a limitation of this approach is a lack of sensitivity to deeper situated tissue structures. Due to the decrease in tissue absorption during the involution process, penetration depth increases 1 to $2 \mathrm{~mm}$ between day 0 and day 237. As a consequence, DOSI becomes slightly more sensitive to the deeper situated tissue structures over time, but we argue that this will only be of marginal influence on the observed relative changes in tissue composition.

The nonparametric model we chose to analyze the longitudinal data requires that data collected at separate spatial points are independent. Though each position represents different tissue volumes, there is some overlap in the interrogation regions for adjacent grid points. Furthermore, there will be some interdependency due to all of the data coming from the same subject. Taking this limitation into account, as well as the fact that this is a case study, these results should not be generalized to a larger population.
Just as breast growth during pregnancy varies substantially among women, ${ }^{30}$ the involution process is likely to depend on individual characteristics. In this case study, two unique characteristics of the participant were the mastectomy of the contralateral breast and the palpable duct in the investigated breast, in which the contrast disappeared gradually along with the involution of the rest of the breast. Other factors of influence on mammary involution may be the total lactation period before cessation, as well as weaning behavior, i.e., abrupt cessation of breastfeeding, versus gradual replacement by supplemental foods. ${ }^{19}$ Although the optical properties of the breast do not seem to significantly correlate with age for premenopausal women, ${ }^{29,33}$ this factor may be of influence on the dynamics of tissue composition during the involution process. Future research with DOSI on mammary involution will enable to investigate the complete range of tissue composition changes and their dependency on these factors of influence.

\subsection{Significance}

This case study demonstrates the potential of DOSI to study lactation physiology by mapping the spatiotemporal changes in tissue composition during mammary involution. Because of the safe levels of optical power and the absence of contrast agents, DOSI is an excellent technique for repeated measurements 
without introducing any health risks. Our data are unique in extent of investigated tissue components (water, lipid, THb, $\mathrm{StO}_{2}$, scattering power, and scattering prefactor), as well as the temporal nature of our measurements. Although Nissan et al. ${ }^{10}$ have carefully studied in vivo fibroglandular tissue changes during mammary involution with MRI, their study only compared mammary tissue composition in the lactating state to the postweaning state. As a consequence, it did not provide any information on the rate of changes in tissue composition.

Knowledge of mammary tissue composition during involution, and also during all other stages of pregnancy and lactation, is highly relevant for breast cancer diagnosis and treatment during these phases. $^{7-9,16}$ The sensitivity of DOSI to changes in $\mathrm{THb}$ concentrations and $\mathrm{StO}_{2}$ levels also offers unique possibilities to study mammary hemodynamics, vascular remodeling, and its relation to milk synthesis, which is a largely unexplored aspect of lactation. ${ }^{34}$ Furthermore, the future study of tissue composition changes during mammary involution itself may provide important insights into its relation to pregnancy-associated breast cancer, because an imbalance in the cell-death pathways that regulate mammary involution can promote mammary tumor genesis. ${ }^{35,36}$

\section{Conclusion}

In conclusion, this case study demonstrates proof of principle that DOSI is an effective and safe tool to investigate lactation physiology. We have used DOSI to monitor involution of the lactating breast, by providing a unique source of information on the temporal changes in the spatial distribution of the mammary lipid, water, and hemoglobin content, as well as its $\mathrm{StO}_{2}$ and tissue microscale structure. For the participant in this case study, we found significant changes in all of those parameters during the involution process, which are consistent with the relatively small amount of literature on this topic. Our findings contribute to the overall knowledge on the process of mammary involution, as well as its consequences for breast cancer diagnosis and treatment after the cessation of breastfeeding.

\section{Disclosures}

The authors declare no competing interests.

\section{Acknowledgments}

We thank the participant of this case study for her time and commitment to the study. Financial support was provided by the National Institutes of Health, Grant Nos. P41EB015890 (Laser Microbeam and Medical Program) and R01-CA142989. Beckman Laser Institute programmatic support from the Arnold and Mabel Beckman Foundation is gratefully acknowledged. The content is solely the responsibility of the authors and does not necessarily represent the official views of the National Institutes of Health. Biostatistics collaboration was provided by the Biostatistics Shared Resource within the Chao Family Comprehensive Cancer Center at the University of California, Irvine, which was supported by the National Institutes of Health (No. P30CA062203).

\section{References}

1. C. G. Victora et al., "Breastfeeding in the 21st century: epidemiology, mechanisms, and lifelong effect," Lancet 387(10017), 475-490 (2016).

2. World Health Organization, and UNICEF, "Global breastfeeding scorecard 2017," 2017, retrieved from www.who.int/nutrition/publications/ infantfeeding/global-bf-scorecard-2017/en/.
3. R. Li et al., "Why mothers stop breastfeeding: mothers' self-reported reasons for stopping during the first year," Pediatrics 122, S69-S76 (2008).

4. S. Lee and S. L. Kelleher, "Biological underpinnings of breastfeeding challenges: the role of genetics, diet, and environment on lactation physiology," Am. J. Physiol. Endocrinol. Metab. 311(2), E405-E422 (2016).

5. D. T. Ramsay et al., "Anatomy of the lactating human breast redefined with ultrasound imaging," J. Anat. 206(6), 525-534 (2005).

6. D. T. Geddes and V. S. Sakalidis, "Ultrasound imaging of breastfeeding-A window to the inside: methodology, normal appearances, and application," J. Hum. Lactation 32(2), 340-349 (2016).

7. A. C. Talele et al., "The lactating breast: MRI findings and literature review," Breast J. 9(3), 237-240 (2003).

8. R. Vashi et al., "Breast imaging of the pregnant and lactating patient: imaging modalities and pregnancy-associated breast cancer," Am. $J$. Roentgenol. 200(2), 321-328 (2013).

9. M. L. Gemignani and D. McCartan, "78 - Carcinoma of the breast in pregnancy and lactation," in The Breast, 5th ed., K. I. Bland et al., Eds., Elsevier, Philadelphia, pp. 993-999.e993 (2018).

10. N. Nissan et al., "Monitoring in-vivo the mammary gland microstructure during morphogenesis from lactation to post-weaning using diffusion tensor MRI," J. Gland Biol. Neoplasia 22(3), 193-202 (2017).

11. S. Jiang et al., "Predicting breast tumor response to neoadjuvant chemotherapy with diffuse optical spectroscopic tomography prior to treatment," Clin. Cancer Res. 20(23), 6006-6015 (2014).

12. B. J. Tromberg et al., "Predicting responses to neoadjuvant chemotherapy in breast cancer: ACRIN 6691 trial of diffuse optical spectroscopic imaging," Cancer Res. 76(20), 5933-5944 (2016).

13. P. Taroni et al., "Non-invasive optical estimate of tissue composition to differentiate malignant from benign breast lesions: a pilot study," Sci. Rep. 7, 40683 (2017).

14. T. D. O'sullivan et al., "Optical imaging correlates with magnetic resonance imaging breast density and revealscomposition changes during neoadjuvant chemotherapy," Breast Cancer Res. 15(1), R14 (2013).

15. R. Choe et al., "Differentiation of benign and malignant breast tumors by in-vivo three-dimensional parallel-plate diffuse optical tomography," J. Biomed. Opt. 14, 024020 (2009).

16. F. Amant et al., "Breast cancer in pregnancy," Lancet 379(9815), 570-579 (2012).

17. C. J. Watson, "Key stages in mammary gland development - Involution: apoptosis and tissue remodelling that convert the mammary gland from milk factory to a quiescent organ," Breast Cancer Res. 8(2), 203-203 (2006).

18. A.-C. Andres and V. Djonov, "The mammary gland vasculature revisited," J. Mammary Gland Biol. Neoplasia 15(3), 319-328 (2010).

19. M. C. Neville et al., "Studies in human lactation: milk volume and nutrient composition during weaning and lactogenesis," Am. J. Clin. Nutr. 54(1), 81-92 (1991).

20. C. O. dos Santos et al., "An epigenetic memory of pregnancy in the mouse mammary gland," Cell Rep. 11(7), 1102-1109 (2015).

21. A. V. Capuco and R. M. Akers, "Mammary involution in dairy animals," J. Mammary Gland Biol. Neoplasia 4(2), 137-144 (1999).

22. I. R. Fleet and M. Peaker, "Mammary function and its control at the cessation of lactation in the goat," J. Physiol. 279(1), 491-507 (1978).

23. F. Bevilacqua et al., "Broadband absorption spectroscopy in turbid media by combined frequency-domain and steady-state methods," Appl. Opt. 39(34), 6498-6507 (2000).

24. A. E. Cerussi et al., "Tissue phantoms in multicenter clinical trials for diffuse optical technologies," Biomed. Opt. Express 3(5), 966-971 (2012).

25. E. Brunner, S. Domhof, and F. Langer, Nonparametric Analysis of Longitudinal Data in Factorial Experiments, J Wiley, New York (2002).

26. B. Brooksby et al., "Imaging breast adipose and fibroglandular tissue molecular signatures by using hybrid MRI-guided near-infrared spectral tomography," Proc. Natl. Acad. Sci. U. S. A. 103(23), 8828-8833 (2006).

27. M. M. El-Sherif and F. Assad, "Changes in some blood constituents of Barki ewes during pregnancy and lactation under semi arid conditions," Small Rumin. Res. 40(3), 269-277 (2001). 
28. H. S. Mitchell and L. Miller, "The effect of age, pregnancy and lactation on the hemoglobin of the albino rat," Am. J. Physiol. Legacy Content 98(2), 311-317 (1931).

29. N. Shah et al., "Spatial variations in optical and physiological properties of healthy breast tissue," J. Biomed. Opt. 9(3), 534-540 (2004).

30. D. B. Cox et al., "Breast growth and the urinary excretion of lactose during human pregnancy and early lactation: endocrine relationships," Exp. Physiol. 84(2), 421-434 (1999).

31. R. Cubeddu et al., "Effects of the menstrual cycle on the red and nearinfrared optical properties of the human breast," Photochem. Photobiol. 72(3), 383-391 (2000).

32. N. Shah et al., "Noninvasive functional optical spectroscopy of human breast tissue," Proc. Natl. Acad. Sci. U. S. A. 98(8), 4420-4425 (2001).

33. T. Durduran et al., "Bulk optical properties of healthy female breast tissue," Phys. Med. Biol. 47(16), 2847-2861 (2002).

34. D. T. Geddes et al., "Blood flow characteristics of the human lactating breast," J. Hum. Lactation 28(2), 145-152 (2012).

35. S. M. McDaniel et al., "Remodeling of the mammary microenvironment after lactation promotes breast tumor cell metastasis," Am. J. Pathol. 168(2), 608-620 (2006).
36. O. C. Rivera, S. R. Hennigar, and S. L. Kelleher, " $\mathrm{ZnT}_{2}$ is critical for lysosome acidification and biogenesis during mammary gland involution," Am. J. Physiol. Regul. Integr. Comp. Physiol. 315(2), R323-R335 (2018).

Nienke Bosschaart is an assistant professor in the Biomedical Photonic Imaging Group at the University of Twente in Netherlands. She received her $\mathrm{PhD}$ from the Academic Medical Center of the University of Amsterdam in 2012. Her research focuses on the development of optical diagnostic tools for mother and child care.

Thomas D. O'Sullivan is an assistant professor in the Department of Electrical Engineering, University of Notre Dame. He received his $\mathrm{PhD}$ in electrical engineering from Stanford University in 2011 and then was a U.S. Department of Defense Breast Cancer Research Program postdoctoral fellow at the University of California, Irvine Beckman Laser Institute (BLI). While at BLI, he also served as a director of the Diffuse Optical Spectroscopy and Imaging Laboratory.

Biographies of the other authors are not available. 